\title{
Dinâmica da comunidade lenhosa de uma floresta de galeria na transição Cerrado-Floresta Amazônica no Leste de Mato Grosso, em um período de sete anos (1999 a 2006)
}

\author{
Aline Miguel', Beatriz, Schwantes Marimon, ${ }^{1,2,3}$, Edmar Almeida de Oliveira ${ }^{2}$, \\ Leandro Maracahipes ${ }^{2}$ \& Ben Hur Marimon-Junior ${ }^{2}$ \\ ${ }^{1}$ Programa de Pós-graduação em Ciências Ambientais, Universidade do Estado de Mato Grosso - UNEMAT, \\ Av. Tancredo Neves, n. 1095, Bairro Cavalhada 2, CEP 78200-000, Cáceres, MT, Brasil \\ ${ }^{2}$ Programa de Pós-graduação em Ecologia e Conservação, \\ Universidade do Estado de Mato Grosso - UNEMAT, Campus de Nova Xavantina, \\ CP 08, CEP 78690-000, Nova Xavantina, MT, Brasil \\ ${ }^{3}$ Autor para correspondência: Beatriz Schwantes Marimon, e-mail: biamarimon@hotmail.com
}

\begin{abstract}
MIGUEL, A., MARIMON, B.S., OLIVEIRA, E.A., MARACAHIPES, L. \& MARIMON-JUNIOR, B.H. Woody community dynamics of a gallery forest in the transition Cerrado-Amazon Forest in Eastern Mato Grosso, over a seven year period (1999 to 2006). Biota Neotrop. 11(1): http://www.biotaneotropica.org.br/v11n1/en/ abstract?article+bn01111012011.
\end{abstract}

\begin{abstract}
The study was carried out on the gallery forest of the Bacaba stream (14 $43^{\prime} 12.2^{\prime \prime} \mathrm{S}$ and $52^{\circ} 21^{\prime} 36.7^{\prime \prime} \mathrm{W}$ ), in the transition between Cerrado and Amazon Forest in Nova Xavantina, Mato Grosso State, Brazil. The objective was to evaluate and compare the dynamics of the woody community at three sections of the forest (upper, middle and lower) in a topographic gradient over a seven year period. The inventory consisted of 141 permanent plots $(10 \times 10 \mathrm{~m})$ distributed in the three sections where all individuals $\geq 15 \mathrm{~cm}$ girth at breast height were recorded in 1999 and 2006. Based in density and basal area, the Periodic Annual Increment (PAI) and dynamics parameters were calculated. In all forest sections the mortality was higher than recruitment. The lower section was the most dynamic, because presented the higher mortality (6.84\%) and recruitment (4.73\%) rates. The community was dynamic, probably due to its current intermediary successional stage and the different environmental conditions depending on the topography. The PAI values of the species in common with all sections confirm differences in increment, indicating that some have restrictions and others benefit to grow in certain environments. On the other hand, some species had similar PAI values in the three forest sections, confirming a plasticity to adapt to different environmental conditions. The species presented different recruitment, mortality and increment rates, related to the ecological groups and topographic position. We suggest that the topographic gradient and seasonal flooding contribute to include this community among the most dynamic tropical forests.

Keywords: forest dynamics, riparian forest, increment, mortality, recruitment.
\end{abstract}

MIGUEL, A., MARIMON, B.S., OLIVEIRA, E.A., MARACAHIPES, L. \& MARIMON-JUNIOR, B.H. Dinâmica da comunidade lenhosa de uma floresta de galeria na transição Cerrado-Floresta Amazônica no Leste de Mato Grosso, em um período de sete anos (1999 a 2006). Biota Neotrop. 11(1): http://www.biotaneotropica. org.br/v11n1/pt/abstract?article+bn01111012011.

Resumo: O estudo foi realizado na floresta de galeria do Córrego Bacaba (14 43' 12,2" S e 52 21' 36,7" W), na transição Cerrado-Floresta Amazônica em Nova Xavantina, Mato Grosso. O objetivo foi avaliar e comparar a dinâmica da comunidade lenhosa de três porções da floresta localizadas em um gradiente topográfico (alto, meio e baixo) em um período de sete anos. O inventário consistiu de 141 parcelas permanentes de $10 \times 10 \mathrm{~m}$ distribuídas nas três porções da floresta, onde foram medidos os indivíduos com CAP $\geq 15$ cm, em 1999 e 2006. Foram calculados os parâmetros de dinâmica e o incremento periódico anual (IPA), com base no número de indivíduos e na área basal. Nas três porções de floresta a mortalidade foi superior ao recrutamento. A porção do baixo foi a mais dinâmica, pois apresentou a maior taxa de mortalidade $(6,84 \%)$ e o maior recrutamento $(4,73 \%)$. A elevada dinâmica da floresta deve-se, provavelmente, ao estágio de sucessão intermediário no qual ela se encontra $\mathrm{e}$ às diferentes condições do ambiente conforme a topografia. Os valores do IPA de algumas espécies comuns às três porções de floresta permitiram confirmar que ocorrem diferenças no incremento de uma porção para outra, indicando que algumas espécies apresentam restrições e outras são beneficiadas para crescer em determinados ambientes. Por outro lado, algumas espécies apresentaram valores de IPA similares nas três porções da floresta, confirmando plasticidade às diferentes condições ambientais. As espécies estudadas apresentaram taxas distintas de recrutamento, mortalidade e incremento relacionadas aos grupos ecológicos e à localização topográfica. Sugere-se que o gradiente topográfico e a inundação sazonal contribuem para posicionar esta comunidade entre as florestas tropicais mais dinâmicas.

Palavras-chave: dinâmica florestal, floresta ripária, incremento, mortalidade, recrutamento. 


\section{Introdução}

O Bioma Cerrado ocupa a porção Central do Brasil, conecta-se com quatro dos seis biomas brasileiros, incluindo a Mata Atlântica, a sudeste, e a Floresta Amazônica, ao norte e é composto de onze fitofisionomias, desde formações campestres mais abertas até formações florestais com dossel fechado, incluindo as florestas de galeria que margeiam os cursos d'água de menor porte (Ribeiro \& Walter 1998).

As florestas do Brasil Central são consideradas inclusões das floras amazônica e atlântica no Bioma Cerrado (Eiten 1994), sendo que, no Estado de Mato Grosso as florestas de galeria apresentam contribuição da flora amazônica mais evidente que no restante do Bioma (Oliveira-Filho \& Ratter 1995, Pinto \& Oliveira-Filho 1999). Neste caso, na porção nordeste do estado, a ligação dos cursos d'água com a Bacia Amazônica pode explicar a elevada representatividade de espécies amazônicas nas fitofisionomias florestais da região (Ratter et al. 1973, Miguel \& Marimon 2008, Marimon et al. 2002, 2003, 2006, 2010).

Além da flora complexa, as florestas ripárias do Cerrado apresentam características particulares em relação ao ambiente físico (e.g. solos e topografia) e às condições biológicas, sendo encraves florestais com importante função na proteção e manutenção dos mananciais hídricos do Bioma (Pinto \& Oliveira-Filho 1999, Silva-Júnior 2005). Além disso, são bastante dinâmicas, com mudanças ocorrendo continuamente em níveis individuais e populacionais ao longo do tempo, mesmo sendo consideradas comunidades estáveis devido ao balanço entre recrutamento e mortalidade (Felfili 1995a).

Os ambientes das florestas de galeria estão associados com pequenos cursos d'água que compõem a rede de drenagem das bacias hidrográficas, exibem forte variação no nível do lençol freático ao longo do ano, em função da topografia, e ocorrem em depressões derivadas de superfícies de erosão recentes, como em sedimentos do Terciário, e outras muito mais antigas, como rochas Pré-Cambrianas (RadamBrasil 1982, Pinto 2002, Marimon et al. 2003). A história geomorfológica complexa contribui para a diversidade nas características topográficas, geológicas e edáficas dos córregos nestas superfícies, que influenciam na diversidade florística e nos parâmetros fitossociológicos das florestas de galeria, conforme a sua localização no relevo, como, na nascente ou proximidade da foz, na margem do curso d'água, no interior da floresta ou na região de contato com outras fitofisionomias (Schiavini 1997, Marimon et al. 2001, 2002, 2003, 2010), este último exercendo o chamado 'efeito de borda', incluindo a entrada ocasional de fogo.

Estas condições de grande variabilidade topográfica e hídrica, de efeito de borda e de alta complexidade florística e fitossociológica podem explicar porque as florestas de galeria normalmente apresentam uma dinâmica florístico-estrutural acentuada ao logo do tempo (Oliveira-Filho et al. 1997, Machado \& Oliveira-Filho 2010). Entretanto, alguns estudos indicam que as florestas de galeria, apesar das flutuações nas taxas de mortalidade e recrutamento, tendem ao equilíbrio dinâmico (Felfili 1995a, b, Oliveira \& Felfili 2005, Pinto \& Hay 2005). Estes estudos buscam compreender, ainda, a estruturação de uma comunidade ou população em um determinado intervalo de tempo, contribuindo para predições sobre o crescimento e a produtividade florestal (Felfili 1995a, Felfili et al. 2000, Lopes \& Schiavini 2007). Contudo, dado o reduzido número de trabalhos sobre dinâmica em florestas de galeria do Brasil Central, ainda existem lacunas a serem preenchidas em relação à ecologia destas florestas.

O objetivo deste trabalho foi avaliar e comparar a dinâmica da comunidade lenhosa de três porções de floresta de galeria localizadas em um gradiente topográfico na zona de transição entre os biomas Cerrado e Floresta Amazônica, no Parque Municipal do Bacaba,
Nova Xavantina, região Leste de Mato Grosso, em um período de sete anos (1999 a 2006). Além disso, o estudo procurou avaliar se a dinâmica da floresta em questão possui padrão diferenciado em relação a outras tipologias florestais ripárias e se a porção de relevo mais plano e próxima da foz (baixo), cujo solo permanece alagado a maior parte do ano, apresenta maior dinâmica em relação às porções com relevo mais acidentado e mais próximas à nascente (alto e meio).

\section{Material e Métodos}

O estudo foi realizado no Parque Municipal do Bacaba, Nova Xavantina, Mato Grosso (Figura 1), em três porções (alto, meio e baixo) da floresta de galeria existente ao longo do Córrego Bacaba (1443'12,2"S e 52 $21^{\circ}$ '36,7'W), distantes cerca de $200 \mathrm{~m}$ entre si, em um gradiente topográfico. As três porções foram selecionadas por estarem dentro dos limites do parque, sendo a porção do alto localizada no limite sul e mais próxima da nascente do Córrego Bacaba, a porção do baixo no limite norte e mais próxima da foz e a porção do meio em uma posição intermediária, a montante de uma pequena barragem de captação da água (Figura 1). A floresta ocupa cerca de $8 \%$ dos quase 500 ha do parque, protege o sistema hídrico do córrego que abastece um bairro residencial do município, e em julho de 2001, foi parcialmente atingida por fogo originado em uma fazenda vizinha, em consequência da prática de renovação do pasto adotada pelos pecuaristas no período da seca.

A altitude média da área é de $346 \mathrm{~m}$ e o clima é do tipo $A w$ de Köppen, com precipitação anual entre 1.300 e 1.500 mm e temperatura média mensal de $25^{\circ} \mathrm{C}$ (Marimon et al. 2003, 2010). A porção do alto apresenta uma declividade média de $42 \%$, com presença de afloramentos rochosos de quartzito e enchentes sazonais com drenagem rápida. $\mathrm{O}$ meio apresenta declividade de aproximadamente $32 \%$, com afloramentos rochosos e lençol freático aflorando no período chuvoso. A porção do baixo apresenta reduzida declividade (cerca de 5\%), drenagem deficiente e ausência de afloramentos rochosos (Marimon et al. 2001, 2002, 2003, 2010).

No ano de 1999 foram estabelecidas 141 parcelas permanentes de $10 \times 10$ m, 47 em cada porção da floresta, visando a caracterização florística e estrutural da vegetação (Marimon et al. 2001, 2002, 2003, 2010). As parcelas foram posicionadas sistematicamente em faixas perpendiculares ao córrego, cobrindo toda área florestada desde a margem até a borda, no contato com o cerrado stricto sensu.

No primeiro inventário (outubro-novembro de 1999), todas as árvores, arvoretas, palmeiras, lianas ou indivíduos mortos em pé com $\mathrm{CAP}$ (circunferência à altura do peito) $\geq 15 \mathrm{~cm}$ foram identificados e medidos a $1,3 \mathrm{~m}$ em relação ao solo e em seguida marcados com uma faixa de tinta amarela (esmalte sintético), de modo a garantir que as medições posteriores fossem efetuadas no mesmo local. No segundo inventário (outubro-novembro de 2006), todos os indivíduos amostrados em 1999 foram remedidos, juntamente com aqueles que atingiram a circunferência mínima de inclusão (recrutas). Foram efetuadas coletas botânicas de todas as espécies e o material testemunho (voucher) encontra-se depositado no Herbário NX da UNEMAT, Campus de Nova Xavantina. As espécies foram classificadas quanto aos grupos ecológicos (pioneira, clímax exigente de luz e clímax tolerante à sombra) de acordo com as características apresentadas pelos indivíduos jovens e adultos e por meio de consultas às bibliografias especializadas (Swaine \& Whitmore 1988, Oliveira-Filho et al. 1994, Pinto \& Hay 2005, Marimon 2005).

Baseando-se no número de indivíduos amostrados em 1999 e em 2006, foram calculadas as taxas de mortalidade, recrutamento, rotatividade (Sheil et al. 1995, 2000), meia-vida e o tempo de duplicação (Lieberman et al. 1985). A estabilidade e o tempo de substituição (turnover time) foram calculados de acordo com Korning \& Balslev 
(1994a, b). O incremento periódico anual (IPA) em diâmetro foi calculado a partir da diferença do DAP (diâmetro a 1,3 m em relação ao solo) no segundo e no primeiro inventário dividido pelo período estudado, em anos (Scolforo 1994, Encinas et al. 2005). Neste caso, tal como proposto por Encinas et al. (2005), o IPA expressa o crescimento anual pela média do crescimento em um determinado período de anos. A partir da área basal foram calculadas as taxas anuais médias de perda e ganho, cujas expressões exponenciais estão descritas em Guimarães et al. (2008), e a rotatividade (Oliveira-Filho et al. 1997). Os parâmetros de dinâmica foram calculados para cada parcela e foram efetuadas comparações pela análise de variância não-paramétrica de Kruskal-Wallis (Sokal \& Rohlf 1995) entre cada porção de floresta. Também foram calculados os parâmetros de dinâmica das principais espécies em VI (valor de importância) em 2006 (Marimon et al. 2002, Miguel \& Marimon 2008). A diferença entre o número de recrutas e mortos em cada porção de floresta foi verificada por comparações entre contagens de Poisson (Zar 1999).

\section{Resultados}

A mortalidade foi superior ao recrutamento nas porções do meio $(Z=2,592, p<0,01)$ e do baixo $(Z=4,423, p<0,001)$, mas não diferiram na porção do alto $(Z=0,602, p>0,5)$, sendo esta a porção que apresentou o menor desbalanceamento entre estes dois parâmetros. Na porção do baixo foi registrada a maior taxa de mortalidade e também a maior taxa de recrutamento, com valores cerca de duas vezes superiores aos do alto e do meio (Tabela 1). Como consequência das elevadas taxas de mortalidade e recrutamento, a porção do baixo apresentou também os menores valores de tempo de meia vida e de duplicação, assim como os menores valores do tempo de substituição e estabilidade e a maior taxa de rotatividade, caracterizando-se como a porção mais dinâmica da floresta estudada (Tabela 1). Na porção do alto, $55 \%$ das espécies registradas entre 1999 e 2006 apresentaram mortalidade, recrutamento ou ambos, no meio foram $69 \%$ e no baixo $70 \%$ das espécies.

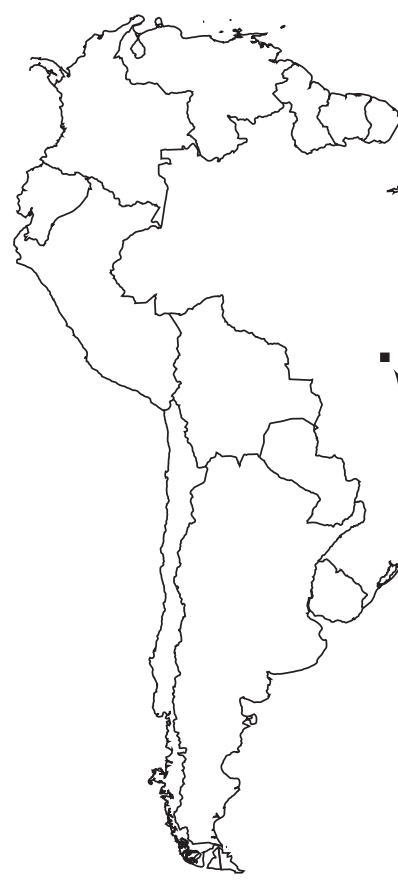

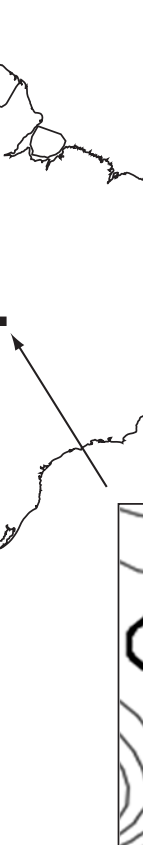

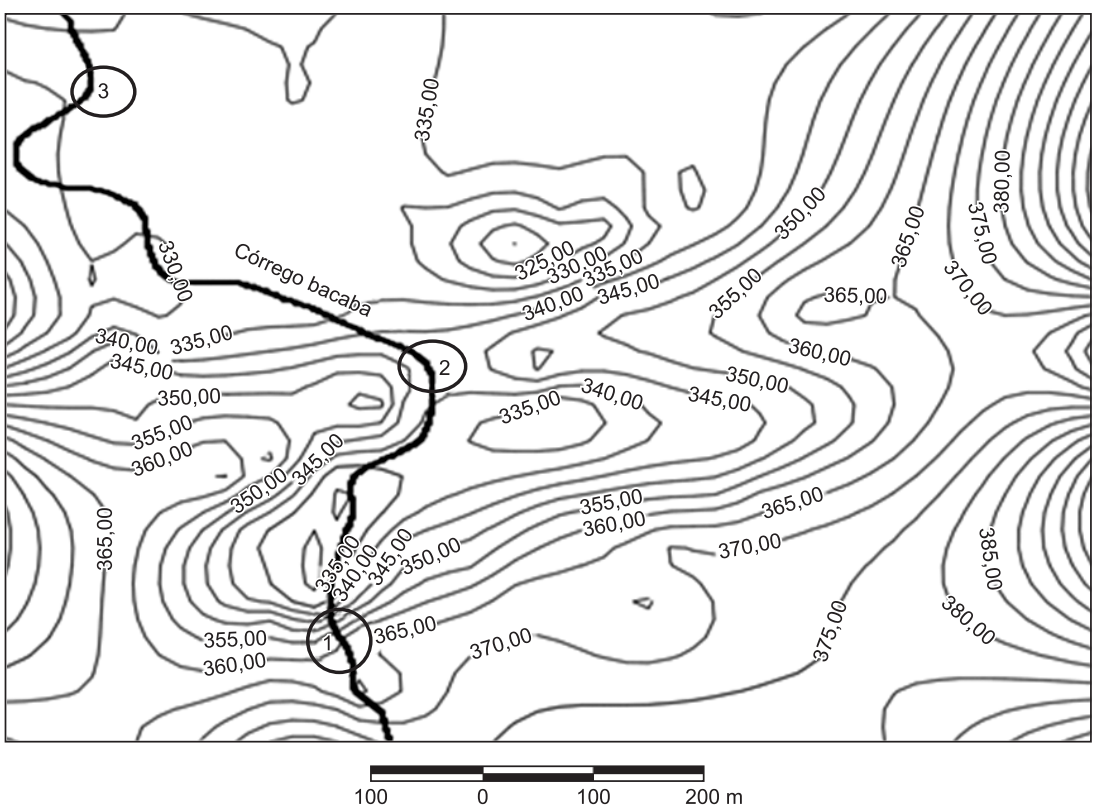

Figura 1. Localização da área de estudo na floresta de galeria do Córrego Bacaba, Nova Xavantina, Mato Grosso, Brasil. Porções do alto (1), meio (2) e baixo (3). As linhas indicam as curvas de nível do terreno e suas respectivas cotas de altitude (m).

Figure 1. Location of the study site at the Bacaba Stream gallery forest, Nova Xavantina, Mato Grosso State, Brazil. Upper (1), middle (2) and lower (3) sections. The lines indicate the contours of the land and their respective elevation (m). 
Foram registradas diferenças entre os grupos ecológicos amostrados nas porções de floresta, tanto em relação ao número de espécies quanto ao número de indivíduos mortos e recrutados (Tabela 2). De modo geral, as espécies clímax exigentes de luz apresentaram a maior porcentagem de espécies e indivíduos mortos e recrutas (Tabela 2). Comparando-se cada grupo ecológico entre as porções da floresta, observou-se que apenas os indivíduos clímax tolerantes à sombra apresentaram percentual de mortalidade superior ao recrutamento (Alto: $Z=1,986, p<0,05$; Meio: $Z=2,179, p<0,01$; Baixo: $Z=3,111, \mathrm{p}<0,001)$.

Considerando-se todas as espécies e indivíduos no período de sete anos (1999 a 2006), o Incremento Periódico Anual (IPA) da comunidade lenhosa em cada porção da floresta variou de 0,20 a $0,35 \mathrm{~cm} \mathrm{ano}^{-1}$ e o maior valor foi registrado para o baixo (Tabela 1). Dentre as espécies avaliadas na floresta do Bacaba, 53\% apresentaram o IPA acima do valor médio da comunidade na porção do alto, $40 \%$ na do meio e $50 \%$ na do baixo. A variação entre os valores de IPA nas três porções da floresta estudada foi elevada, sendo todos os coeficientes de variação superiores a 100\% (Tabela 1).

Quando analisamos os parâmetros de dinâmica das espécies de maior VI (valor de importância) para cada porção da floresta estudada, Astrocaryum vulgare (alto) se destacou entre as de maior taxa de mortalidade e Aspidosperma subincanum (meio) e Virola urbaniana (baixo) apresentaram elevada taxa de recrutamento (Tabela 3).

Tabela 1. Parâmetros de dinâmica da comunidade lenhosa em três porções (alto, meio e baixo) da floresta de galeria do Córrego Bacaba entre 1999 e 2006, Nova Xavantina, Mato Grosso. Sendo: $\mathrm{AB}=$ área basal $\left(\mathrm{m}^{2} \cdot \mathrm{ha}^{-1}\right) \mathrm{e}$ $\mathrm{H}=$ teste de Kruskal-Wallis. ${ }^{*} \mathrm{p}<0,05 \mathrm{e}^{* * *} \mathrm{p}<0,001$.

Table 1. Dynamics parameters of the woody community in three sections (upper, middle and lower) of the gallery forest of the Bacaba stream between 1999 and 2006, Nova Xavantina, Mato Grosso State. AB = basal area $\left(\mathrm{m}^{2}\right.$. $\mathrm{ha}^{-1}$ ) and $\mathrm{H}=$ Kruskal-Wallis test, $* \mathrm{p}<0.05$ and $* * * \mathrm{p}<0,001$.

\begin{tabular}{|c|c|c|c|c|}
\hline Parâmetros & Alto & Meio & Baixo & $\mathbf{H}$ \\
\hline $\begin{array}{l}\text { Taxa de mortalidade } \\
\left(\% \text { ano }^{-1}\right)\end{array}$ & $2,38^{b}$ & $3,72^{\mathrm{b}}$ & $6,84^{\mathrm{a}}$ & $30,48 * * *$ \\
\hline $\begin{array}{l}\text { Taxa de recrutamento } \\
\left(\% \text { ano }^{-1}\right)\end{array}$ & $1,92^{\mathrm{b}}$ & $2,66^{\mathrm{b}}$ & $4,73^{\mathrm{a}}$ & $14,08 * * *$ \\
\hline $\begin{array}{l}\text { Taxa anual de ganho } \\
\left(\% \text { ano }^{-1}\right)\end{array}$ & $3,36^{\mathrm{b}}$ & $3,71^{\mathrm{b}}$ & $6,73^{\mathrm{a}}$ & $20,19 * * *$ \\
\hline $\begin{array}{l}\text { Taxa anual de perda } \\
\left(\% \text { ano }^{-1}\right)\end{array}$ & $1,97^{\mathrm{b}}$ & $2,90^{\mathrm{b}}$ & $4,63^{\mathrm{a}}$ & $20,06 * * *$ \\
\hline Tempo de meia vida (anos) & $29,12^{\mathrm{a}}$ & $18,63^{\mathrm{a}}$ & $10,13^{\mathrm{b}}$ & $22,86 * * *$ \\
\hline Tempo de duplicação (anos) & $36,10^{\mathrm{a}}$ & $26,06^{\mathrm{a}}$ & $14,65^{b}$ & $6,49 *$ \\
\hline $\begin{array}{l}\text { Taxa de rotatividade } \\
\text { (indivíduos, } \% \text { ano }{ }^{-1} \text { ) }\end{array}$ & $2,15^{\mathrm{b}}$ & $3,19^{b}$ & $5,78^{a}$ & $34,10 * * *$ \\
\hline $\begin{array}{l}\text { Taxa de rotatividade } \\
\left(\mathrm{AB}, \% \mathrm{ano}^{-1}\right)\end{array}$ & $2,66^{\mathrm{b}}$ & $3,30^{\mathrm{b}}$ & $5,68^{\mathrm{a}}$ & $23,81 * * *$ \\
\hline $\begin{array}{l}\text { Tempo de substituição } \\
\text { (anos) }\end{array}$ & 32,61 & 22,34 & 12,39 & - \\
\hline Estabilidade (anos) & 6,98 & 7,43 & 4,52 & - \\
\hline $\begin{array}{l}\text { Área Basal de incremento } \\
\left(\mathrm{m}^{2} \cdot \mathrm{ha}^{-1}\right)\end{array}$ & 1,7100 & 1,5314 & 2,2818 & - \\
\hline $\begin{array}{l}\text { Área Basal de decremento } \\
\left(\mathrm{m}^{2} \cdot \mathrm{ha}^{-1}\right)\end{array}$ & 0,3565 & 0,1475 & 0,3545 & - \\
\hline $\begin{array}{l}\text { Área Basal de recrutas } \\
\left(\mathrm{m}^{2} \cdot \mathrm{ha}^{-1}\right)\end{array}$ & 0,2541 & 0,2246 & 1,8087 & - \\
\hline $\begin{array}{l}\text { Incremento periódico anual } \\
\text { (IPA, } \mathrm{cm})\end{array}$ & $0,20^{\mathrm{b}}$ & $0,25^{\mathrm{b}}$ & $0,35^{\mathrm{a}}$ & $42,51 * * *$ \\
\hline $\begin{array}{l}\text { Coeficiente de variação } \\
\text { (IPA) }\end{array}$ & 1,44 & 1,02 & 1,10 & - \\
\hline
\end{tabular}

Dentre as espécies que tiveram o IPA avaliado (Tabela 4), apenas Mabea pohliana, Himatanthus bracteatus e Cordia sellowiana apresentaram valores acima da média de incremento em todas as porções onde ocorreram. Seis espécies apresentaram valores acima da média em duas porções da floresta avaliada e quatro em apenas uma porção (alto: Tetragastris altissima, Licania apetala e Ephedranthus parviflorus e baixo: Ormosia excelsa). As espécies comuns às três porções de floresta e que apresentaram incrementos acima da média das comunidades em pelo menos duas porções caracterizam-se como espécies de dossel, sendo algumas pioneiras. O maior valor absoluto de IPA foi registrado para Tachigali vulgaris L.G. Silva \& H.C. Lima $\left(2,05 \mathrm{~cm}^{\mathrm{ano}} \mathrm{o}^{-1}\right)$, uma espécie pioneira.

\section{Discussão}

Os valores da taxa anual de mortalidade nas três porções da floresta estudada, em geral, foram superiores aos valores registrados por Felfili (1995a), van den Berg (2001), Pinto (2002), Lopes \& Schiavini (2007), Oliveira \& Felfili (2008) e Machado \& Oliveira-Filho (2010). De acordo com Felfili (1995a), valores de taxa de mortalidade próximos a 3,5\% ano $^{-1}$ seriam típicos de áreas que sofreram distúrbios. Este aspecto confirma o padrão dinâmico da comunidade estudada e pode estar relacionado ao fogo que atingiu a floresta em 2001, sendo esta uma hipótese a ser testada. Segundo Lopes \& Schiavini (2007), taxas menores de mortalidade são características de florestas mais estáveis, sem grandes distúrbios e auto-mantenedoras, com populações numericamente constantes em um equilíbrio dinâmico, o que não implica em imobilidade ou incapacidade de mudar ao longo do tempo.

Em comparação com outras florestas de galeria do Brasil Central (Oliveira \& Felfili 2008) as taxas de mortalidade e recrutamento da floresta do Bacaba foram elevadas. Neste caso, tal como observado pelas referidas autoras, os valores elevados de mortalidade e recrutamento levam a uma elevada rotatividade, o que é esperado em ambientes sob intenso efeito de borda e grande variação topográfica, como é o caso da vegetação na área do presente estudo, uma estreita faixa de floresta tropical localizada em uma área de relevo acidentado e circundada por formações savânicas (Miguel \& Marimon 2008, Marimon et al. 2003, 2010).

Apesar de muito próximas geograficamente, as porções estudadas apresentaram diferenças em relação à estrutura (Marimon et al. 2002, 2003) e aos padrões de dinâmica da comunidade lenhosa. Neste caso, a heterogeneidade ambiental (alto = elevada declividade e afloramentos rochosos e baixo = relevo plano, sem rochas e com afloramento do lençol freático) e as perturbações antrópicas (fogo) podem ter contribuído para evidenciar as diferenças registradas, semelhante ao que foi registrado por Machado \& Oliveira-Filho (2010) em uma floresta semidecídua de Minas Gerais e por Lopes \& Schiavini (2007), que estudaram uma floresta de galeria em Minas Gerais e também registraram diferentes parâmetros de dinâmica para comunidades localizadas em áreas próximas.

Embora os valores da taxa de mortalidade tenham sidos elevados nas três porções estudadas, o incremento em área basal sugere que esta floresta encontra-se em um período intermediário de sucessão, onde estaria diminuindo a densidade e aumentando a área basal total (Miguel \& Marimon 2008) em um padrão de auto-desbaste, tal como foi observado por Felfili (1995a) e Werneck et al. (2000). Em uma floresta de galeria no Distrito Federal Oliveira \& Felfili (2005) registraram elevada redução da densidade total e aumento da área basal devido a um maior sombreamento da área, inibindo o crescimento de espécies heliófilas e conduzindo a comunidade a um estágio de sucessão mais climácico. 
Tabela 2. Número de espécies e indivíduos da comunidade lenhosa que apresentaram mortalidade (Mort.) e recrutamento (Recr.) em três porções (alto, meio e baixo) da floresta de galeria do Córrego Bacaba entre 1999 e 2006, Nova Xavantina, Mato Grosso. Sendo: Clímax luz = espécies clímax exigentes de luz e Clímax sombra $=$ espécies clímax tolerantes à sombra, $\chi^{2}=$ teste de qui-quadrado, $* P<0,05, * * P<0,01, * * * P<0,001$ e ns não significativo. Valores percentuais entre parênteses.

Table 2. Number of species and individuals of the woody community with mortality (Mort.) and recruitment (Recr.) in three sections (upper, middle and lower) of the gallery forest of the Bacaba stream between 1999 and 2006, Nova Xavantina, Mato Grosso State. Clímax luz = light demanding climax species and Clímax sombra $=$ Shade-tolerant climax species, $\chi^{2}=$ chi-square test, ${ }^{*} P<0.05, * * P<0.01, * * * P<0.001$ and ${ }^{\text {ns }}$ not significant. $\%$ between parentheses.

\begin{tabular}{|c|c|c|c|c|c|c|}
\hline \multirow[t]{2}{*}{ Grupo Ecológico } & \multicolumn{2}{|c|}{ Alto } & \multicolumn{2}{|c|}{ Meio } & \multicolumn{2}{|c|}{ Baixo } \\
\hline & Mort. & Recr. & Mort. & Recr. & Mort. & Recr. \\
\hline \multicolumn{7}{|l|}{ Espécies } \\
\hline Pioneiras & $8(27,4)$ & $4(14,3)$ & $8(17,4)$ & $6(18,2)$ & $8(16,7)$ & $11(28,2)$ \\
\hline Clímax luz & $11(37,8)$ & $15(53,6)$ & $23(50,0)$ & $17(51,5)$ & $29(60,4)$ & $21(53,8)$ \\
\hline Clímax sombra & $10(34,8)$ & $9(32,1)$ & $14(32,6)$ & $10(30,3)$ & $11(22,9)$ & $7(18,0)$ \\
\hline$\chi^{2}$ & $0,483^{\mathrm{ns}}$ & $6,502 *$ & $7,603^{*}$ & $5,836^{*}$ & $16,126 * * *$ & $8,001 *$ \\
\hline \multicolumn{7}{|l|}{ Indivíduos } \\
\hline Pioneiras & $16(24,6)$ & $14(25,4)$ & $15(15,3)$ & $13(19,4)$ & $89(42,3)$ & $58(40,0)$ \\
\hline Clímax luz & $23(35,4)$ & $24(43,6)$ & $44(44,9)$ & $32(47,8)$ & $85(40,4)$ & $73(50,3)$ \\
\hline Clímax sombra & $26(40,0)$ & $17(31,0)$ & $39(39,8)$ & $22(32,8)$ & $36(17,3)$ & $14(9,7)$ \\
\hline$\chi^{2}$ & $2,433^{\mathrm{ns}}$ & $2,874^{\mathrm{ns}}$ & $14,714 * * *$ & $8,101^{*}$ & $24,887 * * *$ & $38,923 * * *$ \\
\hline
\end{tabular}

Tabela 3. Parâmetros de dinâmica das espécies com maior VI (Falor de Importância) em três porções (alto, meio e baixo) da floresta de galeria do Córrego Bacaba entre 1999 e 2006, Nova Xavantina, Mato Grosso. Sendo: $\mathrm{m}=$ taxa de mortalidade $\left(\%\right.$ ano $\left.{ }^{-1}\right), \mathrm{r}=$ taxa de recrutamento $\left(\%\right.$ ano $\left.{ }^{-1}\right), \mathrm{t}_{1 / 2}=$ tempo de meia vida $($ anos $)$, $\mathrm{t}_{2}=$ tempo de duplicação (anos), TRo $=$ taxa de rotatividade (indiv., $\%$ ano $\mathrm{o}^{-1}$ ), TRoAB $=$ taxa de rotatividade (área basal, $\%$ ano ${ }^{-1}$ ), Sub. $=$ tempo de substituição $\left(\right.$ anos), Est. $=$ estabilidade $\left(\right.$ anos), $\mathrm{ABin}=$ área basal de incremento $\left(\mathrm{m}^{2} \cdot \mathrm{ha}^{-1}\right), \mathrm{Abd}=$ área basal de decremento $\left(\mathrm{m}^{2} \mathrm{ha}^{-1}\right)$ e Abr $=$ área basal de recrutas $\left(\mathrm{m}^{2} \cdot\right.$ ha $\left.\mathrm{a}^{-1}\right)$.

Table 3. Dynamics parameters of species with higher VI (importance value) in three sections (upper, middle and lower) of the gallery forest of the Bacaba

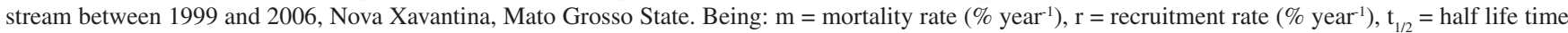
(years), $\mathrm{t}_{2}=$ doubling time (years), TRo $=$ turnover rate (indiv., $\%$ year ${ }^{-1}$ ), TRoAB $=$ turnover rate (basal area, $\%$ year ${ }^{-1}$ ), Sub. $=$ time of replacement $($ years), Est. $=$ stability (years), ABin $=$ increment basal area $\left(\mathrm{m}^{2} \mathrm{ha}^{-1}\right), \mathrm{Abd}=$ decrement basal area $\left(\mathrm{m}^{2} \cdot \mathrm{ha}^{-1}\right)$ e Abr $=$ recruits basal area $\left(\mathrm{m}^{2}\right.$. ha $\left.\mathrm{a}^{-1}\right)$.

\begin{tabular}{|c|c|c|c|c|c|c|c|c|c|c|c|}
\hline Espécies & m & $\mathbf{r}$ & $t_{1 / 2}$ & $\mathbf{t}_{\mathbf{2}}$ & TRo & TRoAB & Sub. & Est. & ABin & Abd & Abr \\
\hline \multicolumn{12}{|l|}{ Alto } \\
\hline Astrocaryum vulgare Mart. & 21,48 & 2,63 & 27,94 & 26,35 & 2,55 & 12,64 & 27,14 & 1,59 & 0,0779 & 0,2153 & 0,0750 \\
\hline Diospyros guianensis (Aubl.) Gürke & 2,27 & 1,66 & 30,53 & 41,75 & 1,96 & 10,95 & 36,14 & 11,22 & 0,1256 & 0,0151 & 0,0118 \\
\hline Calophyllum brasiliense Cambess. & 0 & 0 & - & - & 0 & 11,92 & - & - & 0,1254 & 0,0008 & 0 \\
\hline Mabea pohliana (Benth.) Müll. Arg. & 1,49 & 2,82 & 46,51 & 24,57 & 2,15 & 8,84 & 35,54 & 21,94 & 0,0540 & 0 & 0,0133 \\
\hline Protium heptaphyllum (Aubl.) Marchand & 2,42 & 1,66 & 28,64 & 41,75 & 2,04 & 9,66 & 35,19 & 13,11 & 0,0665 & 0,0349 & 0,0075 \\
\hline Licania blackii Prance & 0,98 & 1,88 & 70,72 & 36,86 & 1,42 & 8,81 & 48,38 & 44,67 & 0,8582 & 0 & 0,0071 \\
\hline Oenocarpus distichus Mart. & 0 & 2,02 & 0 & 34,31 & 1,01 & 11,92 & 34,31 & 34,31 & 0,2782 & 0,0002 & 0,0185 \\
\hline Tetragastris altissima (Aubl.) Swart. & 4,69 & 0 & 14,77 & - & 2,34 & 13,25 & 7,38 & 14,77 & 0,0474 & 0,0002 & 0 \\
\hline \multicolumn{12}{|l|}{ Meio } \\
\hline Aspidosperma subincanum Mart. & 1,49 & 4,89 & 46,51 & 14,17 & 3,19 & 8,59 & 30,34 & 32,34 & 0,0691 & 0 & 0,0235 \\
\hline Tetragastris altissima (Aubl.) Swart. & 2,07 & 2,97 & 33,48 & 23,33 & 2,52 & 12,51 & 28,4 & 10,15 & 0,0475 & 0 & 0,0086 \\
\hline Apuleia leiocarpa (Vogel) J.F. Macbr. & 0 & 0 & - & - & 0 & 15,35 & 0 & 0 & 0,0584 & 0,0018 & 0 \\
\hline Pouteria gardneri (Mart. \& Miq.) Baehni & 1,05 & 1,13 & 66,01 & 61,34 & 1,09 & 10,97 & 63,67 & 4,67 & 0,0366 & 0 & 0,0018 \\
\hline Ephedranthus parviflorus S. Moore & 3,13 & 2,07 & 22,14 & 33,48 & 2,6 & 8,31 & 27,81 & 11,34 & 0,0442 & 0,0055 & 0,0113 \\
\hline Protium heptaphyllum (Aubl.) Marchand & 1,49 & 1,49 & 46,51 & 46,51 & 1,49 & 9,75 & 46,51 & 0 & 0,0303 & 0 & 0,0046 \\
\hline Luehea candicans Mart. \& Zucc. & 3,75 & 2,02 & 18,48 & 34,31 & 2,88 & 5,9 & 26,39 & 15,83 & 0,0218 & 0,0187 & 0,0036 \\
\hline \multicolumn{12}{|l|}{ Baixo } \\
\hline Mauritia flexuosa L. f. & 1,84 & 3,44 & 37,67 & 20,14 & 2,64 & 9,24 & 28,90 & 17,53 & 0,0604 & 0,0660 & 1,1446 \\
\hline Astrocaryum vulgare Mart. & 1,13 & 5,05 & 61,34 & 13,72 & 3,09 & 8,52 & 37,53 & 47,62 & 0,1021 & 0,1058 & 0,1499 \\
\hline Virola urbaniana Warb. & 10,08 & 10,08 & 6,87 & 6,87 & 10,08 & 2,98 & 6,87 & 0 & 0,1524 & 0 & 0,0814 \\
\hline Physocalymma scaberrimum Pohl. & 0 & 1,05 & - & 66,01 & 0,52 & 10,55 & 33,00 & 66,01 & 0,0832 & 0,0031 & 0,0045 \\
\hline Himatanthus bracteatus (A.DC.) Woodson & 0,73 & 5,86 & 94,95 & 11,82 & 3,29 & 3,73 & 53,38 & 83,13 & 0,1831 & 0,0002 & 0,0451 \\
\hline Tapirira guianensis Aubl. & 13,82 & 6,02 & 5,01 & 11,51 & 9,92 & 5,31 & 8,26 & 6,5 & 0,2579 & 0,0183 & 0,0367 \\
\hline Mabea pohliana (Benth.) Müll. Arg. & 2,97 & 5,88 & 23,33 & 11,78 & 4,42 & 4,83 & 17,55 & 11,55 & 0,0673 & 0,0010 & 0,0362 \\
\hline Xylopia aromatica (Lam.) Mart. & 5,07 & 1,29 & 13,67 & 53,73 & 3,18 & 3,83 & 33,7 & 40,06 & 0,1942 & 0,0012 & 0,0065 \\
\hline Aspidosperma subincanum Mart. & 0 & 0,76 & - & 91,20 & 0,38 & 5,49 & 45,6 & 91,20 & 0,1296 & 0 & 0,0021 \\
\hline Ormosia excelsa Benth. & 0 & 0,98 & - & 70,72 & 0,49 & 8,35 & 35,36 & 70,72 & 0,0956 & 0,0025 & 0,0051 \\
\hline
\end{tabular}


Tabela 4. Incremento periódico anual de diâmetro (IPA, $\left.\mathrm{cm} \mathrm{ano}^{-1}\right)$ e coeficiente de variação (CV, \%) por espécie em três porções (alto, meio e baixo) da floresta de galeria do Córrego Bacaba, entre 1999 e 2006, Nova Xavantina-MT. CV calculado para as espécies representadas por no mínimo 10 indivíduos.

Table 4. Periodic annual increment of diameter (IPA, $\mathrm{cm} \mathrm{year}^{-1}$ ) and coefficient of variation (CV, \%) per species in three sections (upper, middle and lower) of the gallery forest of the Bacaba stream between 1999 and 2006, Nova Xavantina, Mato Grosso State. CV calculated for species represented by at least 10 individuals.

\begin{tabular}{|c|c|c|c|c|c|c|}
\hline \multirow[t]{2}{*}{ Espécies } & \multicolumn{2}{|c|}{ Alto } & \multicolumn{2}{|c|}{ Meio } & \multicolumn{2}{|c|}{ Baixo } \\
\hline & IPA & $\mathrm{CV}$ & IPA & CV & IPA & CV \\
\hline Mabea pohliana (Benth.) Müll. Arg. & 0,23 & 1,00 & 0,35 & 0,78 & 0,36 & 0,74 \\
\hline Aspidosperma subincanum Mart. & 0,15 & 1,66 & 0,26 & 0,63 & 0,52 & 0,38 \\
\hline Protium heptaphyllum (Aubl.) Marchand & 0,23 & 0,92 & 0,16 & 0,87 & 0,36 & - \\
\hline Tetragastris altissima (Aubl.) Swart. & 0,21 & 0,93 & 0,20 & 0,82 & 0,24 & - \\
\hline Ephedranthus parviflorus S. Moore & 0,21 & 0,42 & 0,21 & 0,66 & - & - \\
\hline Himatanthus bracteatus (Aubl.) Swart. & 0,24 & 0,77 & 0,30 & - & 0,63 & 0,54 \\
\hline Licania apetala (E. Mey) Fritsch & 0,41 & 0,65 & 0,23 & - & 0,22 & - \\
\hline Licania blackii Prance & 0,34 & 0,77 & 0,30 & - & 0,21 & - \\
\hline Calophyllum brasiliense Cambess. & 0,30 & 0,84 & 0,43 & - & 0,15 & - \\
\hline Diospyros guianensis (Aubl.) Gürke & 0,18 & 0,86 & 0,20 & - & - & - \\
\hline Luehea candicans Mart. \& Zucc. & 0,16 & - & 0,19 & 1,00 & 0,15 & - \\
\hline Apuleia leiocarpa (Vogel) J.F. Macbr. & 0,20 & - & 0,20 & 0,94 & 0,25 & - \\
\hline Unonopsis lindmanii R. E. Fr. & - & - & 0,25 & 0,66 & - & - \\
\hline Pouteria gardneri (Mart. \& Miq.) Baehni & 0,22 & - & 0,20 & 0,64 & 0,50 & - \\
\hline Cordia sellowiana Cham. & 0,21 & - & - & - & 0,62 & 0,58 \\
\hline Virola urbaniana Warb. & 0,02 & - & 0,72 & - & 0,62 & 0,72 \\
\hline Ormosia excelsa Benth. & - & - & - & - & 0,38 & 0,75 \\
\hline
\end{tabular}

A maior taxa de mortalidade em relação ao recrutamento, assim como a taxa de ganho em área basal superior à perda, registradas no presente estudo, podem representar um quadro de instabilidade, com acúmulo de biomassa em pé e declínio da densidade de árvores, associado a uma fase de construção intermediária a tardia do ciclo-silvigenético pós-distúrbio (Chagas et al. 2001, OliveiraFilho et al. 2007, Machado \& Oliveira-Filho 2010). Lopes \& Schiavini (2007) afirmam que clima, geologia, incidência luminosa e distúrbios naturais favorecem mudanças, uma vez que as alterações que provocam a saída também podem favorecer a entrada de espécies mais adaptadas às condições ambientais da comunidade. Estudos em longo prazo são essenciais, inclusive para avaliar se as alterações na comunidade estão relacionadas a mudanças globais (climáticas, antrópicas, aumento nos níveis atmosféricos de $\mathrm{CO}_{2}$, radiação solar, etc.) visto que diversos estudos têm demonstrado o aumento na biomassa e produtividade primária de florestas da região amazônica nas últimas décadas (Nemani et al. 2003, Laurance et al. 2004, Arias et al. 2010).

O alto foi a porção que apresentou o menor percentual de espécies para as quais foram registradas mortalidade, recrutamento ou ambos, sendo esta caracterizada como a porção mais estável da floresta. Felfili (1995b) observou que $86 \%$ de todas as espécies de uma floresta de galeria sofreram mortalidade, recrutamento ou ambos em um período de seis anos, conferindo um caráter de elevada dinâmica àquela vegetação. Por outro lado, em florestas mais estáveis e que sofrem menos perturbações, as espécies de maior abundância apresentam poucas mudanças na densidade (Felfili et al. 2000, Appolinário et al. 2005).

O tempo de meia vida e de duplicação e a taxa de rotatividade e estabilidade nas três porções da floresta estudada também confirmam o padrão dinâmico em comparação com outras florestas no Bioma Cerrado (Pinto 2002, Appolinário et al. 2005, Paiva et al. 2007, Oliveira \& Felfili 2008). No presente estudo, a maior dinâmica foi registrada para a porção do baixo em função das altas taxas de entrada e saída (recrutamento e mortalidade). De acordo com Korning \& Balslev (1994b), a dinâmica de uma comunidade pode ser expressa pelo tempo de substituição e pela taxa de rotatividade, que sintetizam as mudanças na floresta (recrutamento ou acréscimo e mortalidade ou decréscimo). Neste caso, assim como a taxa de rotatividade, a elevada mortalidade também pode estar associada ao fogo e ao maior período de encharcamento do solo na porção do baixo (Marimon et al. 2003, Miguel \& Marimon 2008) e o elevado recrutamento pode ter sido favorecido pela entrada de luz difusa no local, fato confirmado pela elevada ocorrência de recrutas de espécies heliófilas nesta porção. Padrão similar em florestas de galeria também foi registrado por van den Berg (2001) e Pinto (2002). No caso da floresta do Bacaba o fogo pode ter sido responsável pela exclusão de algumas espécies sensíveis e pela redução no número de indivíduos (Felfili et al. 2000, Aquino et al. 2007) e o encharcamento sazonal pressupõem adaptações fisiológicas particulares das espécies para competir em ambientes extremos (Scarano et al. 1997). Entretanto, estudos adicionais são necessários para confirmar este padrão na floresta estudada.

Apenas as espécies clímax tolerantes à sombra apresentaram mortalidade superior ao recrutamento em todas as porções da floresta estudada, indicando que a estrutura da comunidade sofrerá mudanças com o passar dos anos. Este fato difere do que foi registrado por Felfili (1995b) e Chagas et al. (2001), onde este grupo apresentou maior recrutamento em relação à mortalidade. Em um padrão similar ao registrado na floresta do presente estudo, Vaccaro (2002), trabalhando em uma floresta estacional, registrou maior taxa de mortalidade nas espécies secundárias tardias. No caso da floresta do Bacaba, é possível que a abertura do dossel, permitindo maior entrada de luz, tenha promovido neste grupo sucessional de espécies um aumento na mortalidade em relação ao recrutamento. Desta forma a elevada dinâmica da floresta do Bacaba, evidenciada pelos elevados valores de mudanças, pode ser também devido ao estágio de sucessão intermediário no qual ela se encontra. 
Em uma floresta de vale em Mato Grosso, investigada por Pinto (2002), os valores da área basal do incremento $\left(0,09\right.$ a $\left.0,4 \mathrm{~m}^{2} \mathrm{ha}^{-1}\right)$ e dos recrutas $\left(0,028\right.$ a $\left.0,14 \mathrm{~m}^{2} \mathrm{ha}^{-1}\right)$ foram inferiores aos da floresta de galeria do Bacaba. Como a floresta estudada pelo referido autor localiza-se em uma depressão (escarpa) de aproximadamente $70 \mathrm{~m}$ em relação às áreas adjacentes, as espécies que ocorrem nesta situação estão sujeitas a uma condição ambiental mais extrema (acentuado gradiente topográfico), quando comparadas à floresta do presente estudo, fato este que pode reduzir o incremento e limitar o recrutamento de muitas espécies.

Astrocaryum vulgare se destacou entre as espécies de maior taxa de mortalidade na porção do alto e, como ela é uma espécie pioneira (Miguel \& Marimon 2008), a redução na densidade desta população pode indicar que a comunidade está em fase de recuperação pós-distúrbio, tal como discutido anteriormente. Aspidosperma subincanum (meio), classificada por Miguel \& Marimon (2008) como clímax tolerante à sombra e Virola urbaniana (baixo), como clímax exigente de luz, apresentaram elevada taxa de recrutamento, indicando que espécies de diferentes grupos ecológicos estão progredindo no estágio sucessional, provavelmente em função das características ambientais de cada porção de floresta. Considerando-se as três porções, Tapirira guianensis esteve entre as espécies mais dinâmicas na floresta estudada. Em uma floresta de galeria em Minas Gerais, Lopes \& Schiavini (2007) também registraram esta espécie entre as de maior mortalidade e recrutamento.

Na floresta do Bacaba, algumas espécies apresentaram parâmetros de dinâmica similares em diferentes porções da floresta, destacando-se Aspidosperma subincanum (meio e baixo) e Mabea pohliana (alto e baixo), evidenciando a maior plasticidade ambiental das mesmas. Outras, como Astrocaryum vulgare e Tetragastris altissima, apresentaram maior mortalidade em uma porção e maior recrutamento em outra. Neste caso, confirma-se o que também foi observado por Pinto (2002), de que os parâmetros de dinâmica das populações podem ser extremamente heterogêneos, sugerindo que o processo de dinâmica é multidirecional. Além disso, Felfili (1997) e van den Berg (2001) observaram que a dinâmica das populações apresenta variações em áreas submetidas a diferentes regimes de distúrbio ou diferentes condições ambientais, como é o caso das três porções de floresta aqui avaliadas. Lieberman (1996) observou que a variação na dinâmica das populações é uma característica intrínseca das espécies tropicais e pode ser um dos mecanismos responsáveis pela coexistência de várias espécies, tal como ocorre na floresta estudada.

Os valores do Incremento Periódico Anual (IPA) da comunidade lenhosa em cada porção da floresta estudada foram próximos e até superiores aos registrados por Oliveira \& Felfili (2008) para uma floresta de galeria no Brasil Central $\left(0,21-0,26 \mathrm{~cm} \mathrm{ano}^{-1}\right)$ e por Pinto (2002), em uma floresta de vale em Mato Grosso $\left(0,21 \mathrm{~cm} \mathrm{ano}^{-1}\right)$. Silva et al. (2002) avaliaram 272 árvores em uma floresta de terra firme na Amazônia central e encontraram incremento médio de $0,16 \mathrm{~cm}^{2} \mathrm{no}^{-1}$ e Barreto \& Uhl (1993) estudaram uma floresta no Pará, registrando valores entre 0,2 e $0,6 \mathrm{~cm}^{a n o}{ }^{-1}$.

Os elevados valores de IPA no presente estudo estão de acordo com o que foi observado por Oliveira \& Felfili (2008), que relacionam as taxas mais elevadas deste parâmetro aos ambientes mais perturbados e com predomínio de espécies pioneiras, as quais apresentam estratégia de crescimento acelerado (Hartshorn 1990) e são adaptadas a condições mais abertas e sob efeito de borda, como normalmente ocorre nas florestas de galeria. De acordo com as referidas autoras, taxas próximas a $0,20 \mathrm{~cm}^{2} \mathrm{ano}^{-1}$, como foi o caso das porções do alto e do meio na floresta do Bacaba, estariam associadas a florestas tropicais não perturbadas, sendo que taxas superiores a $0,3 \mathrm{~cm}$ ano $^{-1}$ seriam típicas de ambientes mais perturbados, como foi o caso da porção do baixo.
Analisando o IPA das espécies de maior VI, destaca-se Aspidosperma subincanum, que apresentou diferença de até $250 \%$ no incremento entre uma porção da floresta para outra, fato que pode indicar restrições de crescimento desta espécie em determinados ambientes e favorecimento em outros. Por outro lado, algumas espécies como Mabea pohliana, Tetragastris altissima e Ephedranthus parviflorus, apresentaram valores de IPA similares em todas as porções onde ocorreram, sugerindo certa plasticidade para se adaptar às variações ambientais. Todas as espécies comuns às três porções de floresta e que apresentaram incrementos acima da média das comunidades em pelo menos duas porções caracterizam-se como espécies de dossel, sendo algumas pioneiras. Felfili (1995a) observou que as espécies que exigem luz e ocupam o dossel superior apresentam taxa de incremento maior e Oliveira \& Felfili (2008) observaram que o rápido crescimento de espécies pioneiras permite a este grupo apresentar uma vantagem competitiva em relação às de estágio secundário de sucessão.

O maior valor absoluto de IPA foi registrado para Tachigali vulgaris, que apresenta elevado crescimento sob condições de maior luminosidade (Felfili et al. 2000, Miguel \& Marimon 2008). Felfili \& Silva-Júnior (1998) observaram que frequentemente são encontrados, para esta espécie, indivíduos de grande porte e mortos em pé. Assim, estes indivíduos, após a queda, promovem a abertura de novas clareiras, mantendo a possibilidade de regeneração e crescimento da espécie em um tipo de círculo virtuoso de auto-favorecimento. Além disso, esta espécie possui também um grande potencial para recuperação de áreas degradadas e reflorestamento, pois apresenta rápido crescimento, elevada desrama e acentuada queda foliar, o que possibilita rápida formação de serapilheira (Alvino et al. 2007), condição fundamental para a recuperação da fertilidade do solo.

As taxas de incremento registradas para as espécies da floresta estudada também foram bastante variáveis, apresentando diferenças entre indivíduos e espécies. Elevado coeficiente de variação nos incrementos das espécies lenhosas é uma característica frequentemente encontrada em florestas tropicais, como consequência da elevada heterogeneidade genética e ambiental típica destes ambientes (Felfili 1995a, Hubbell et al. 1999). Neste caso, a variação dos incrementos entre os indivíduos de uma mesma espécie pode indicar um potencial de reação às mudanças ambientais (Marimon 2005), onde espécies com elevada plasticidade ambiental tendem a apresentar elevados coeficientes de variação para o incremento, tal como foi o caso de Aspidosperma subincanum no presente estudo. Por outro lado, a competição intraespecífica e a exigência por luz (competição por interferência) estariam entre as principais causas da variabilidade em florestas de galeria, sugerindo que os indivíduos que precisam de luz para crescer podem permanecer com o incremento igual ou próximo de zero até que ocorra a abertura de uma clareira (Felfili 1995a, b), como pode ser o caso de Virola urbaniana na porção do alto.

Tal como registrado no presente estudo, Protium heptaphyllum também apresentou valores de incremento superiores à média da comunidade em uma floresta de galeria no Distrito Federal (Oliveira \& Felfili 2008). As referidas autoras observaram que esta espécie, não pioneira e tolerante à sombra, cresce rapidamente sob condições naturais. Licania apetala, que apresentou incremento abaixo da média da comunidade em duas porções avaliadas no presente estudo, também apresentou incrementos abaixo da média na floresta estudada por Oliveira \& Felfili (2008). Estes aspectos confirmam que muitas espécies podem apresentar padrões de crescimento similares, independentemente de sua distribuição geográfica no Bioma Cerrado.

As diferenças registradas na dinâmica da floresta de galeria do Bacaba nos levam a destacar a variabilidade em que os processos de dinâmica operam dentro de uma mesma comunidade e o grau 
de heterogeneidade espacial que estes processos produzem ao longo do tempo (Pinto 2002, Lopes \& Schiavini 2007). Assim, para compreender plenamente a dinâmica da vegetação em comunidades que apresentam elevadas variações internas, como foi o caso das três porções geograficamente próximas da floresta do Córrego Bacaba, são necessárias seguidas remedições e também a investigação de particularidades dos microsítios analisados, como fatores microclimáticos, edáficos e hídricos derivados das diferentes condições topográficas em cada porção da floresta (Marimon et al. 2003).

Os resultados do presente estudo indicam uma aplicação prática na recomendação de espécies que podem ser utilizadas em iniciativas de recomposição de florestas de galeria degradadas na região e, em longo prazo, modelar o comportamento das espécies em relação a prováveis mudanças climáticas. Neste caso, a seleção de espécies pioneiras ou exigentes de luz que apresentam simultaneamente reduzida taxa de mortalidade e elevadas taxas de recrutamento e incremento pode ser uma alternativa para o plantio em áreas em estágios iniciais de sucessão. Da mesma forma, espécies tolerantes à sombra com reduzida mortalidade e elevado potencial de recrutamento e incremento podem ser utilizadas para o plantio em etapas posteriores.

Concluímos que a comunidade estudada é um exemplo de floresta tropical altamente dinâmica, provavelmente devido ao estresse sofrido em função das diferentes condições ambientais ao longo do gradiente topográfico e ao afloramento do lençol freático, que causa o encharcamento sazonal do solo e pode estar determinando a condição de maior dinâmica na porção do baixo. As espécies das três porções de floresta apresentaram distintas taxas de recrutamento, mortalidade e incremento, que podem estar relacionadas aos grupos ecológicos, ao posicionamento no dossel e às condições ambientais de cada porção da floresta.

\section{Agradecimentos}

À CAPES pela bolsa concedida à primeira autora e à FAPEMAT (Projeto: Tecnologia de bases ecológicas para a recomposição, uso sustentável de florestas de galeria e proteção de recursos hídricos, Proc. $\left.\mathrm{N}^{\circ} 0738 / 2006\right)$ pelo auxílio financeiro.

\section{Referências Bibliográficas}

ALVINO, F.O., SILVA NETO, P.A., MUNIZ, A.L.V. \& RIBEIRO M.S. 2007. Armazenamento e germinação de sementes de Sclerolobium paniculatum Vogel (Leguminosae-Caesalpinioideae). R. Bras. Bioci. 5(2):726-728.

APPOLINÁRIO, V., OLIVEIRA-FILHO, A.T. \& GUILHERME, F.A.G. 2005. Tree population and community in a Brazilian tropical semideciduous forest. Rev. Bras. Bot. 28(2):347-360.

AQUINO, F.G., WALTER, B.M.T. \& RIBEIRO, J.F. 2007. Woody community dynamics in two fragments of cerrado stricto sensu over seven-year period (1995-2002), MA, Brazil. Rev. Bras. Bot. 30(1):113-121.

ARIAS, P.A., FU, R., HOYOS, C.D., LI, W. \& ZHOU, L. 2010. Changes in cloudiness over the Amazon rainforests during last two decades: diagnostic and potential causes. Clim. Dyn. Online First ${ }^{\mathrm{TM}}$. DOI 10.1007/ s00382-010-0903-2.

BARRETO, P. \& UHL, C.O. 1993. O potencial de produção sustentável da madeira em Paragominas-Pará na Amazônia Oriental. Considerações ecológicas e econômicas. In I Congresso Florestal Pan-Americano e VII Congresso Florestal Brasileiro (SBS/SBEF, orgs.). SBS, Curitiba, p.387-392.

CHAGAS, R.K., OLIVEIRA-FILHO, A.T., VAN DEN BERG, E. \& SCOLFORO, J.R.S. 2001. Dinâmica de populações arbóreas em um fragmento de Floresta Estacional Semidecidual Montana em Lavras, Minas Gerais. Rev. Árvore 25(1):39-57.
EITEN, G. 1994. Vegetação do cerrado. In Cerrado: caracterização, ocupação e perspectivas (M.N. Pinto, org.). Editora UnB, Brasília, p.17-73.

ENCINAS, J.I., SILVA, G.F. \& PINTO, J.R.R. 2005. Idade e crescimento das árvores. EdUnB, Brasília, DF., v. 7. Comunicações Técnicas Florestais.

FELFILI, J.M. 1995a. Growth, recruitment and mortality in the Gama gallery forest in central Brazil over a six-year period (1985-1991). J. Trop. Ecol. 11:67-83.

FELFILI, J.M. 1995b. Diversity, structure and dynamics of a gallery forest in central Brazil. Vegetatio 117:1-15.

FELFILI, J.M. 1997. Dynamics of the natural regeneration in the Gama gallery forest in Central Brazil. Forest Ecol. Manag. 91:235-245.

FELFILI, J.M. \& SILVA-JÚNIOR, M.C. 1998. Distribuição dos diâmetros numa faixa de cerrado na Fazenda Água Limpa (FAL) em Brasília-DF. Acta Bot. Bras. 2:85-105

FELFILI, J.M., REZENDE, A.V., SILVA JÚNIOR, M.C. \& SILVA, M.A. 2000. Changes in the floristic composition of cerrado sensu strict in Brazil over a nine-year period. J. Trop. Ecol. 16:579-590.

GUIMARÃES, J.C.C., VAN DEN BERG, E., CASTRO, G.C., MACHADO, E.L.M. \& OLIVEIRA-FILHO, A.T. 2008. Dinâmica do componente arbustivo-arbóreo de uma floresta de galeria aluvial no planalto de Poços de Caldas, MG, Brasil. Rev. Bras. Bot. 31(4):621-632.

HARTSHORN, G.S. 1990. An overview of neotropical forest dynamics. In Four Neotropical Rainforests (A.H. Gentry, ed.). Yale University, New Haven, p. 585-600.

HUBBELL, S.P., FOSTER, R.P., O'BRIEN, S.T., HARMS, K.E., CONDIT, R., WECHSLER, B., WRIGHT, S.J. \& LAO, S.L. 1999. Light gaps disturbance, recruitment limitations and tree diversity in a neotropical forest. Science 283:554-557.

KORNING, J. \& BALSLEV, H. 1994a. Growth and mortality of trees in Amazonian tropical rain forest in Ecuador. J. Veg. Sci. 4:77-86.

KORNING, J. \& BALSLEV, H. 1994b. Growth rates and mortality patterns of tropical lowland tree species and the relation to forest in Amazonian Ecuador. J. Trop. Ecol. 10:151-166.

LAURANCE, W.F., OLIVEIRA, A.A., LAURANCE, S.G., CONDIT, R., NASCIMENTO, H.E.M., SANCHEZ-THORIN, A.C., LOVEJOY, T.E., ANDRADE, A., D'ANGELO, S., RIBEIRO, J.E. \& DICK, C.W. 2004. Pervasive alteration of tree communities in undisturbed Amazonian forests. Nature 428(6979):171-175.

LIEBERMAN, D. 1996. Demography of tropical tree seedlings: a review. In The ecology of tropical tree seedlings (M.D. Swaine, ed.). UNESCO, Paris, p.131-138.

LIEBERMAN, D., LIEBERMAN, M., PERALTA, R. \& HARTSHORN, G. 1985. Mortality patterns and stand turnover rates in a wet tropical forest in Costa Rica. J. Ecol. 73:915-924.

LOPES, S.F. \& SCHIAVINI, I. 2007. Dinâmica da comunidade arbórea de mata de galeria da Estação Ecológica do Panga, Minas Gerais, Brasil. Acta Bot. Bras. 21(2):249-261.

MACHADO, E.L.M. \& OLIVEIRA-FILHO, A.T. 2010. Spatial patterns of tree community dynamics are detectable in a small (4 ha) and disturbed fragment of the Brazilian Atlantic forest. Acta Bot. Bras. 24(1):150-161.

MARIMON, B.S. 2005. Dinâmica de uma floresta monodominante de Brosimum rubescens Taub. e comparação com uma floresta mista em Nova Xavantina-MT. Tese de Doutorado, Universidade de Brasília, Brasília.

MARIMON, B.S., FELFILI, J.M., LIMA, E.S. \& RODRIGUES, A.J. 2001. Distribuição de circunferências e alturas em três porções da mata de galeria do Córrego Bacaba, Nova Xavantina-MT. Rev. Árvore 25(3):335-343.

MARIMON, B.S., FELFILI, J.M. \& LIMA, E.S. 2002. Floristic and phytosociology of the gallery forest of the Bacaba stream, Nova Xavantina, Mato Grosso, Brasil. Edinb. J. Bot. 59(2):303-318.

MARIMON, B.S., FELFILI, J.M., LIMA, E.S. \& PINHEIRO-NETO, J. 2003. Padrões de distribuição de espécies na Mata de Galeria do Córrego Bacaba, Nova Xavantina, Mato Grosso, em relação a fatores ambientais. B. Herb. Ezechias Paulo Heringer 12(1):1-10. 
MARIMON, B.S., FELFILI, J.M., LIMA, E.S. \& MARIMON-JUNIOR, B.H. 2010. Environmental determinants for natural regeneration of gallery forest at the Cerrado/Amazonia boundaries in Brazil. Acta Amaz. 40:107-118.

MARIMON, B.S., LIMA, E.S., DUARTE, T.G., CHIEREGATTO, L.C. \& RATTER, J.A. 2006. Observations on the vegetation of northeastern Mato Grosso, Brazil. IV. An analysis of the Cerrado-Amazonian Forest ecotone. Edinb. J. Bot. 63(2-3):323-341.

MIGUEL, A. \& MARIMON, B.S. 2008. Mudanças na composição florística e na diversidade de espécies em três áreas da mata de galeria do Córrego Bacaba (Nova Xavantina, MT). In Gestão e educação ambiental: água, biodiversidade e cultura (J.E. Santos \& C. Galbiati, orgs.). Ed. Rima, São Carlos, v.1., p.93-116.

NEMANI, R., KEELING, C.D., HASHIMOTO, H., JOLLY, W., PIPER, S., TUCKER, C., MYNENI, R. \& RUNNING, S. 2003. Climate-driven increases in Global Terrestrial Net Primary Production from 1982 to 1999. Science 300:1560-1563.

OLIVEIRA, E.C.L. \& FELFILI, J.M. 2005. Estrutura e dinâmica da regeneração natural de uma mata de galeria no Distrito Federal, Brasil. Acta Bot. Bras. 19(4):801-811.

OLIVEIRA, A.P. \& FELFILI, J.M. 2008. Dinâmica da comunidade arbórea de uma mata de galeria do Brasil Central em um período de 19 anos (1985-2004). Rev. Bras. Bot. 31(4):597-610.

OLIVEIRA-FILHO, A.T. \& RATTER, J.A. 1995. A study of the origin of central Brazilian forests by the analysis of plant species distribution patterns. Edinb. J. Bot. 52(2):141-194.

OLIVEIRA-FILHO, A.T., MELLO, J.M. \& SCOLFORO, J.R.S. 1997. Effects of past disturbance and edges on tree community structure and dynamics within a fragment of tropical semideciduous forest in southeastern Brazil over a five year period (1987-1992). Plant Ecology 131:45-66.

OLIVEIRA-FILHO, A.T., CARVALHO, W.A.C., MACHADO, E.L.M., HIGUCHI, P., APPOLINÁRIO, V., CASTRO, G.C., SILVA, A.C., SANTOS, R.M., BORGES, L.F., CORREA, B.S. \& ALVES, J.M. 2007. Dinâmica da comunidade e populações arbóreas da borda e interior de um remanescente florestal na Serra da Mantiqueira, Minas Gerais, em um intervalo de cinco anos (1999-2004). Rev. Bras. Bot. 30(1):149-161.

OLIVEIRA-FILHO, A.T., VILELA, E.A., CARVALHO, D.A. \& GAVILANES, M.L. 1994. Effect of soil and topography on the distribution of tree species in a tropical riverine forest in south-eastern Brazil. J. Trop. Ecol. 10:483-508.

PAIVA, L.V., ARAÚJO, G.M. \& PEDRONI, F. 2007. Structure and dynamics of woody plant community of tropical semi-deciduous seasonal forest in the "Estação Ecológica do Panga", municipality of Uberlândia, Minas Gerais, Brazil. Rev. Bras. Bot. 30(3):365-373.

PINTO, J.R.R. 2002. Dinâmica da comunidade arbórea-arbustiva em uma floresta de vale no Parque Nacional da Chapada dos Guimarães, Mato Grosso. Tese de Doutorado, Universidade de Brasília, Brasília.

PINTO, J.R.R. \& OLIVEIRA-FILHO, A.T. 1999. Perfil florístico da comunidade arbórea de uma floresta de vale no Parque Nacional da Chapada dos Guimarães, Mato Grosso, Brasil. Rev. Bras. Bot. 22(1):53-67.
PINTO, J.R.R. \& HAY, J.D.V. 2005. Mudanças florísticas e estruturais na comunidade arbórea de uma floresta de vale no Parque Nacional da Chapada dos Guimarães, Mato Grosso, Brasil. Rev. Bras. Bot. 28(3):523-539.

RADAMBRASIL. 1982. Levantamento de recursos naturais. Ministério das Minas e Energia, Brasília, Folha SD 21, Cuiabá.

RATTER, J.A., RICHARDS, P.W., ARGENT, G. \& GIFFORD, D.R. 1973. Observations on the vegetation of northeastern Mato Grosso, I. The woody vegetation types of the Xavantina-Cachimbo Expedition area. Philos. T. Roy. Soc. B. 226:449-492.

RIBEIRO, J.F. \& WALTER, B.M.T. 1998. Fitofisionomias do bioma Cerrado. In Cerrado: ambiente e flora (S.M. Sano \& S.P. Almeida, eds.). EmbrapaCPAC, Planaltina, p.89-166.

SCARANO, F.R., RIBEIRO, K.T., MORAES, L.F.D. \& LIMA, H.C. 1997. Plant establishment on flooded and unflooded patches of a freshwater swamp forest in southeastern Brazil. J. Trop. Ecol. 14:793-803.

SCHIAVINI, I. 1997. Environmental characterization and groups of species in gallery forests. In International Symposium on assessment and monitoring of forests in tropical dry regions with special reference to gallery forests (J. Imaña-Encinas \& C. Kleinn, eds.). EdUnB, Brasília, p.107-113.

SCOLFORO, J.R. 1994. Mensuração florestal. ESAL/FAEPE, Lavras.

SHEIL, D., BURSLEM, D.F.R.P. \& ALDER, D. 1995. The interpretation of mortality rates measures. J. Trop. Ecol. 83:331-333.

SHEIL, D., JENNINGS, S. \& SAVILL, P. 2000. Long-term permanent plot observations of vegetation dynamics in Budongo, a Ugandan rain forest. J. Trop. Ecol. 16:765-800.

SILVA, R.P., SANTOS, J., TRIBUZY, E.S., CHAMBERS, J.Q., NAKAMURA, S. \& HIGUCHI, N. 2002. Diameter increment and growth patterns for individual tree growing in Central Amazon, Brazil. Forest Ecol. Manag. 166:295-301.

SILVA-JÚNIOR, M.C. 2005. Fitossociologia e estrutura diamétrica na Mata de Galeria do Pitoco, na Reserva Ecológica do IBGE, DF. Cerne 11(2):147-158.

SOKAL, R.R. \& ROHLF, F.J. 1995. Biometry. Freeman \& Co., New York.

SWAINE, M.D. \& WHITMORE, T.C. 1988. On the definition of ecological species groups in tropical rain forests. Vegetatio 75:81-86.

VACCARO, S. 2002. Crescimento de uma Floresta Estacional Decidual, em três estágios sucessionais, no Município de Santa Tereza, RS, Brasil. Tese de Doutorado, Universidade Federal de Santa Maria, Santa Maria.

VAN DEN BERG, E. 2001. Variáveis ambientais e dinâmica estrutural e populacional de uma floresta de galeria em Itutinga, MG. Tese de Doutorado, UNICAMP, Campinas.

WERNECK, M.S., FRANCESCHINELLI, E.V. \& TAMEIRÃO-NETO, E. 2000. Mudanças na florística e estrutura de uma floresta decídua durante um período de quatro anos (1994-1998), na região do Triângulo Mineiro, MG. Rev. Bras. Bot. 23(4):401-413.

ZAR, J.H. 1999. Biostatistical analysis. Prentice Hall, New Jersey. 